\title{
HALF-BROTHERS IN THE SCHULHOF FAMILY?
}

\author{
David VoKrouhlickÝ ${ }^{1}$ AND DaVID NesVornÝ ${ }^{2}$ \\ ${ }^{1}$ Institute of Astronomy, Charles University, V Holešovičkách 2, CZ-18000 Prague 8, Czech Republic; vokrouhl@cesnet.cz \\ ${ }^{2}$ Southwest Research Institute, 1050 Walnut Street, Suite 300, Boulder, CO 80302, USA; davidn@boulder.swri.edu \\ Received 2010 November 19; accepted 2011 May 3; published 2011 June 13
}

\begin{abstract}
Disruptive collisions in the asteroid belt produced groups of fragments known as the asteroid families. The studies of identified asteroid families help us to better understand issues related to impact physics, space weathering, asteroid interior, and collisional evolution of the main belt. Here, we analyze a family near the central main belt asteroid (2384) Schulhof. We show that the previously found group of objects around (81337) 2000 GP36 is actually a sub-cluster in the larger Schulhof family. Using backward integrations we demonstrate that the orbits of sub-cluster asteroids converge to that of (2384) Schulhof at $780 \pm 100 \mathrm{kyr}$ ago, suggesting that the breakup event happened very recently. Interestingly, a similar analysis of the two newly discovered members of the Schulhof family may indicate a second event $\lesssim 100 \mathrm{kyr}$ ago (e.g., secondary collision, fission, satellite instability). If confirmed, the formation history of the Schulhof family would suggest that small asteroids may have very colorful lifetimes. Additional astrometric observations of the two new member asteroids will be needed to improve their present orbit and better constrain their past histories.
\end{abstract}

Key words: minor planets, asteroids: general

\section{INTRODUCTION}

Efficient all sky surveys have produced a wealth of new astrometric and photometric data over the past decade. This trend not only continues, but accelerates with new programs such as PanSTARRS and LSST. As a result, the research on asteroid families, products of catastrophic breakups in the asteroid belt, is presently flourishing. While the information about previously known families is being significantly completed, new lines of research are also being developed. For example, the new data led to the identification of very young families. This research direction opened about a decade ago with the discovery of the Karin family (Nesvorný et al. 2002; see also Nesvorný et al. 2003; Novaković 2010). More recently, families younger than $1 \mathrm{Myr}$ have been discovered as clusters in the space of osculating - rather than traditional proper-orbital elements (Nesvorný et al. 2006a, 2008; Nesvorný \& Vokrouhlický 2006; Pravec \& Vokrouhlický 2009). Discoveries of young asteroid families find important applications in studies of the structures of the distribution of interplanetary dust (e.g., Nesvorný et al. 2006b, 2006c; Vokrouhlický et al. 2008; Espy et al. 2009), spikes in the accretion of interplanetary dust onto the terrestrial planets (e.g., Farley et al. 2006), and space weathering (e.g., Jedicke et al. 2004; Vernazza et al. 2009; Willman et al. 2010; see also Chapman 2004 and Gaffey 2010 for general discussion of space weathering).

In this paper, we continue analysis of the population of very young asteroid families in the main belt. We focus on a case that has an interesting relation to the previous work. Pravec \& Vokrouhlický (2009) noted a statistically significant cluster of three small asteroids around (81337) 2000 GP36 and showed that they are likely members of a new young family. However, they missed the existence of another cluster of three small objects around a nearby asteroid (2384) Schulhof, which is reported here for the first time. We show that the two clusters actually form a single family that most likely originated from a large cratering impact on (2384) Schulhof. According to our analysis the impact happened in the past million years. Interestingly, the two parts of the Schulhof family-one around
Schulhof and the other around 2000 GP36-are curiously separated in orbital space, a situation not yet seen in the young asteroid families. We analyze possible causes of this separation and suggest different possibilities of solution, with future observations being able to distinguish between them.

\section{SCHULHOF FAMILY}

\subsection{Basic Information}

(2384) Schulhof is a small asteroid in the central part of the main belt. Its estimated absolute magnitude ranges from 11.7 (MPC) to 12.2 (AstOrb), thus reflecting a characteristic $\sim 0.5$ mag uncertainty. Assuming a geometric albedo of 0.15 (consistent with the mean albedo value of small nearby asteroids; e.g., Nathues 2010), one obtains a size range between 12.5 and $15.7 \mathrm{~km}$. Including the albedo uncertainty of $\simeq 0.05$, the size range would become $10.5-18.5 \mathrm{~km}$. These minimum and maximum size values provide an estimate of the escape velocity from the surface of this asteroid. We obtain values between 5 and $10 \mathrm{~m} \mathrm{~s}^{-1}$, depending on the bulk density and triaxiality assumptions. Ditteon et al. (2002) have determined that Schulhof has relatively fast rotation with a period of $\sim 3.294 \mathrm{hr}$ (observations taken in 2002 March and April) and about half a magnitude amplitude of the light curve. Data from the Sloan Digital Sky Survey (SDSS; Ivezić et al. 2001; Parker et al. 2008) show that (2384) Schulhof has colors with a principal component $\mathrm{PC}_{1} \simeq 0.137 \pm 0.034$ (formal error), roughly an average value of S-type asteroids (e.g., Parker et al. 2008). Synthetic proper orbital elements of Schulhof provided by the AstDyS site (Knežević et al. 2002) are $a_{\mathrm{P}}=2.6099 \mathrm{AU}$ (semimajor axis), $e_{\mathrm{P}}=0.1622$ (eccentricity), and $\sin i_{\mathrm{P}}=0.2304$ (sine of inclination), which allows us to identify (2384) Schulhof as a member of the broad Eunomia family (e.g., Mothé-Diniz et al. 2005). The S-type spectral classification of Schulhof matches the predominant type in the Eunomia family (e.g., Lazzaro et al. 1999; Nathues 2010).

The immediate vicinity of (2384) Schulhof contains three small (1-2 km size) asteroids forming a local cluster (Tables 1 and 2). Using the simple positive-definite quadratic metrics 
Table 1

Osculating Equinoctical Orbital Elements, Their Uncertainties, and Other Parameters of Members of the Schulhof Family

\begin{tabular}{|c|c|c|c|c|c|c|c|c|}
\hline Asteroid & & $\begin{array}{c}a \\
(\mathrm{AU})\end{array}$ & $h$ & $k$ & $p$ & $q$ & $\begin{array}{c}\lambda \\
(\mathrm{deg})\end{array}$ & $\begin{array}{c}H \\
(\mathrm{mag})\end{array}$ \\
\hline \multirow[t]{4}{*}{2384} & Schulhof & 2.61061406 & -0.06708836 & -0.10152809 & 0.01662864 & 0.11750890 & 175.91928 & 11.7 \\
\hline & 2007 EV68 & 2.61022037 & -0.06641219 & -0.10069380 & 0.01668393 & 0.11755620 & 114.31349 & 17.4 \\
\hline & 2008 RA126 & 2.61136572 & -0.06743607 & -0.10033786 & 0.01774666 & 0.11734098 & 168.87176 & 16.7 \\
\hline & 2009 EL11 & 2.60816230 & -0.06512061 & -0.10421552 & 0.01670985 & 0.11790526 & 285.50627 & 17.1 \\
\hline \multicolumn{9}{|c|}{ Formerly 81337 cluster } \\
\hline \multirow[t]{4}{*}{81337} & 2000 GP36 & 2.61205271 & -0.05865443 & -0.10390022 & 0.02146948 & 0.11781403 & 347.48314 & 14.6 \\
\hline & 2001 UR193 & 2.60889467 & -0.06039002 & -0.10363756 & 0.01976410 & 0.11779578 & 44.15196 & 15.6 \\
\hline & 2003 FK6 & 2.60944124 & -0.05754619 & -0.10285965 & 0.02481475 & 0.11722200 & 101.85305 & 16.1 \\
\hline & 2008 GW33 & 2.60963123 & -0.05787958 & -0.10357731 & 0.02719033 & 0.11728482 & 32.73977 & 18.1 \\
\hline \multicolumn{9}{|c|}{ Uncertainty $(\delta a, \delta h, \delta k, \delta p, \delta q, \delta \lambda)$} \\
\hline \multirow[t]{4}{*}{2384} & Schulhof & $2.0 \mathrm{e}-8$ & $8.1 \mathrm{e}-8$ & $1.3 \mathrm{e}-7$ & $7.2 \mathrm{e}-8$ & $1.1 \mathrm{e}-7$ & $1.2 \mathrm{e}-5$ & $\ldots$ \\
\hline & 2007 EV68 & $5.9 \mathrm{e}-4$ & $2.5 \mathrm{e}-4$ & $1.7 \mathrm{e}-4$ & $1.1 \mathrm{e}-5$ & $1.3 \mathrm{e}-4$ & $1.1 \mathrm{e}-1$ & $\ldots$ \\
\hline & 2008 RA126 & $1.3 \mathrm{e}-3$ & $1.9 \mathrm{e}-3$ & $2.1 \mathrm{e}-3$ & $1.1 \mathrm{e}-4$ & $3.2 \mathrm{e}-4$ & $4.1 \mathrm{e}-1$ & $\cdots$ \\
\hline & 2009 EL11 & $2.5 \mathrm{e}-5$ & $1.3 \mathrm{e}-4$ & $1.0 \mathrm{e}-4$ & $4.7 \mathrm{e}-7$ & $8.9 \mathrm{e}-6$ & $1.9 \mathrm{e}-2$ & $\cdots$ \\
\hline \multicolumn{9}{|c|}{ Formerly 81337 cluster } \\
\hline \multirow[t]{4}{*}{81337} & $2000 \mathrm{GP} 36$ & $1.0 \mathrm{e}-7$ & $1.6 \mathrm{e}-7$ & $2.7 \mathrm{e}-7$ & $1.3 \mathrm{e}-7$ & $2.0 \mathrm{e}-7$ & $3.9 \mathrm{e}-5$ & $\cdots$ \\
\hline & 2001 UR193 & $1.1 \mathrm{e}-6$ & $3.6 e-7$ & $3.7 \mathrm{e}-6$ & $3.7 \mathrm{e}-7$ & $7.2 \mathrm{e}-7$ & $3.2 \mathrm{e}-4$ & $\cdots$ \\
\hline & 2003 FK6 & $3.0 \mathrm{e}-7$ & $4.6 e-7$ & $8.3 e-7$ & $2.2 \mathrm{e}-7$ & $5.5 \mathrm{e}-7$ & $6.0 \mathrm{e}-5$ & $\cdots$ \\
\hline & 2008 GW33 & $2.1 \mathrm{e}-3$ & $9.6 e-4$ & $8.1 \mathrm{e}-4$ & $9.6 e-5$ & $4.2 \mathrm{e}-4$ & $1.4 \mathrm{e}-1$ & $\ldots$ \\
\hline
\end{tabular}

Notes. Osculating orbital elements and their uncertainty for epoch MJD 55400 as calculated by the OrbFit9 software (http://newton.dm.unipi.it/orbfit/). We use a heliocentric equinoctical system of non-singular elements: $a$ is the semimajor axis, $(h, k)=e(\sin \varpi, \cos \varpi)$, where $e$ is the eccentricity and $\varpi$ is the longitude of perihelion, $(p, q)=\tan (i / 2)(\sin \Omega, \cos \Omega)$, where $i$ is the inclination and $\Omega$ is the longitude of node, and $\lambda=\varpi+M$ is the mean longitude in orbit ( $M$ is the mean anomaly). The default reference system is that of the mean ecliptic of J2000. The absolute magnitude values $H$ were taken from AstDyS site. Note that the uncertainty in $H$ may be as large as 0.5 mag, especially for single-opposition objects 2007 EV68, 2008 RA126, and 2008 GW33.

Table 2

Osculating Keplerian Orbital Elements of Members of the Schulhof Family

\begin{tabular}{llccccccc}
\hline \hline Asteroid & \multicolumn{1}{c}{$\begin{array}{c}a \\
(\mathrm{AU})\end{array}$} & $e$ & $\begin{array}{c}i \\
(\mathrm{deg})\end{array}$ & $\begin{array}{c}\Omega \\
(\mathrm{deg})\end{array}$ & $\begin{array}{c}\omega \\
(\mathrm{deg})\end{array}$ & $\begin{array}{c}M \\
(\mathrm{deg})\end{array}$ & $\begin{array}{c}H \\
(\mathrm{mag})\end{array}$ \\
\hline 2384 & Schulhof & 2.610614 & 0.1216915 & 13.53638 & 8.0544 & 205.4017 & 322.4631 & 11.7 \\
& 2007 EV68 & 2.610469 & 0.1205871 & 13.53945 & 8.0772 & 205.2939 & 260.8714 & 17.4 \\
& 2008 RA126 & 2.611366 & 0.1208939 & 13.53589 & 8.6002 & 205.3044 & 314.9671 & 16.7 \\
& 2009 EL11 & 2.608159 & 0.1228623 & 13.58349 & 8.0659 & 203.9237 & 73.5552 & 17.1 \\
\hline \multicolumn{7}{c}{ Formerly } & 81337 cluster \\
\hline 81337 & 2000 GP36 & 2.612052 & 0.1193127 & 13.65778 & 10.3279 & 199.1180 & 138.0371 & 14.6 \\
& 2001 UR193 & 2.608893 & 0.1199556 & 13.62258 & 9.5241 & 200.7035 & 193.9237 & 15.6 \\
& 2003 FK6 & 2.609442 & 0.1178628 & 13.66516 & 11.9523 & 197.2729 & 252.6277 & 16.1 \\
& 2008 GW33 & 2.608006 & 0.1193880 & 13.74160 & 13.1771 & 196.1020 & 183.4923 & 18.1 \\
\hline
\end{tabular}

Notes. Osculating orbital elements for epoch MJD 55400 were calculated by the OrbFit9 software (http://newton.dm.unipi.it/orbfit/). The first four objects have orbits very similar to that of (2384) Schulhof, including the longitude of node $\Omega$ and argument of pericenter $\omega$, and form a sub-cluster. The last four objects reside on orbits that have systematically larger $\Omega$ values and smaller $\omega$ values than (2384) Schulhof. They form a separate sub-cluster in the Schulhof family that was previously noted by Pravec \& Vokrouhlický (2009).

$d_{\mathrm{NV}}$ in five-dimensional space of osculating orbital elements (Nesvorný \& Vokrouhlický 2006, their Equation (1)), we find that $d_{\mathrm{NV}} \leqslant 40 \mathrm{~m} \mathrm{~s}^{-1}$ for all these objects. We roughly estimate that there is $\lesssim 10^{-4}$ likelihood that the cluster of objects near (2384) Schulhof is a random fluctuation in the distribution of Eunomia family members. ${ }^{3}$

\footnotetext{
3 We used formula (5) from Vokrouhlický \& Nesvorný (2008), $n=4$, and several nested surrounding zones about Schulhof to define the background density of asteroids and see how it affects the probability estimate. We typically had 1000-2500 asteroids in the background zone (defining $N$ ) and 60,000 to more than a million of dividing cells of the five-dimensional space of osculating orbital elements (defining $M$ ). The choice of our cells also reflected orbital uncertainty, especially since two of the three asteroids surrounding (2384) Schulhof have been observed during a single opposition only (Table 1).
}

Objects in the cluster around (81337) 2000 GP36, previously noted by Pravec \& Vokrouhlický (2009), are also located a small distance from Schulhof $\left(60 \mathrm{~m} \mathrm{~s}^{-1} \lesssim d_{\mathrm{NV}} \lesssim\right.$ $110 \mathrm{~m} \mathrm{~s}^{-1}$; Tables 1 and 2). Few other asteroids have a $d_{\mathrm{NV}} \lesssim$ $150 \mathrm{~m} \mathrm{~s}^{-1}$ distance from (2384) Schulhof. The proximity of the two asteroid clusters prompted us to investigate their possible relation. To that end we first considered nominal (bestfit) orbits of all asteroids with $d_{\mathrm{NV}} \lesssim 150 \mathrm{~m} \mathrm{~s}^{-1}$ relative to (2384) Schulhof and propagated their orbits backward in time for $2 \mathrm{Myr}$. This test allowed us to reach two conclusions.

1. All asteroids other than those in the Schulhof and 2000 GP36 sub-clusters reside on orbits that diverge from 
that of (2384) Schulhof in the past and/or have significantly different mean orbital eccentricity and inclination values.

2. Asteroids in the Schulhof and 2000 GP36 sub-clusters show good convergence 4 of secular angles (longitude of node and pericenter) toward the orbit of (2384) Schulhof during the integrated period of time: those about Schulhof converge nominally at $\sim 100 \mathrm{kyr}$ ago, while those in the 2000 GP36 cluster converge nominally at $\sim 700 \mathrm{kyr}$ ago. An in-depth description of our convergence analysis and age estimate is reported in Section 2.3.

The first result indicates that several asteroids with $d_{\mathrm{NV}} \lesssim$ $150 \mathrm{~m} \mathrm{~s}^{-1}$ relative to (2384) Schulhof are interlopers, whose distance from Schulhof, if expressed in the three-dimensional space of mean orbital elements, is hundreds of $\mathrm{m} \mathrm{s}^{-1}$ (inserting mean orbital elements instead of the osculating elements in $d_{\mathrm{NV}}$ and neglecting differences in secular angles; see Rożek et al. 2011 for an alternate approach). The Schulhof family is embedded in a dense zone of the Eunomia family, and thus the existence of background objects can be expected.

\subsection{Proper Elements for Schulhof Family}

To substantiate previous results, we selected the eight candidate members of the Schulhof family from Tables 1 and 2, and a few nearest asteroids in the osculating element space (with $d_{\mathrm{NV}}$ smaller than $\left.125 \mathrm{~m} \mathrm{~s}^{-1}\right),{ }^{5}$ and integrated their nominal orbits forward in time for $10 \mathrm{Myr}$. We then used the technique described in Knežević et al. (2002) and determined their synthetic proper elements. These results allowed us to confirm the above-mentioned conclusion drawn from the mean orbital elements, namely, that the candidate bodies in the Schulhof family also form a tight cluster in the proper element space. From the surrounding asteroids, we identified only one additional singleopposition object—2008 EK72 — whose proper orbital elements are close to the Schulhof cluster. A closer inspection, however, indicates that this object may belong to the background population rather than the Schulhof family, because the secular angles of its nominal orbit do not show past convergence to the orbit of (2384) Schulhof. This result will significantly improve in the future, when the orbit of 2008 EK72 will become less uncertain by its recovery. At this time, we drop this body and other identified interlopers from further analysis.

Focusing now on the eight selected bodies in the Schulhof family (Tables 1 and 2), we complemented our previous integration by taking 19 clones of each of the objects. The clones all started in the respective uncertainty ellipsoid in the osculating element space around each of the nominal orbits. We propagated their orbits for $10 \mathrm{Myr}$ and computed synthetic proper elements for each of the clones. Distribution of the proper elements determined for the clones helped us to estimate the uncertainty of the proper orbital elements.

The proper elements of the Schulhof family are shown in Figure 1. Note that the proper elements for asteroids residing on multi-opposition orbits (black symbols) show a tight clustering around the orbit of (2384) Schulhof which is tighter than their clustering in osculating elements. Specifically, all multiopposition members in Table 1 have $d_{\text {prop }} \sim 5.5 \mathrm{~m} \mathrm{~s}^{-1}$ from

\footnotetext{
4 Convergence of secular angles toward the orbit of (2384) Schulhof means that their values relative to those of the Schulhof orbit become significantly smaller than they are today (several degrees difference; see Table 2).

5 We used $d_{\mathrm{NV}}<125 \mathrm{~m} \mathrm{~s}^{-1}$ to select all asteroids that are orbitally similar to (2384) Schulhof. Things are not sensitive to the exact value of this cutoff since asteroids with $d_{\mathrm{NV}}>125 \mathrm{~m} \mathrm{~s}^{-1}$ are unrelated to the family.
}

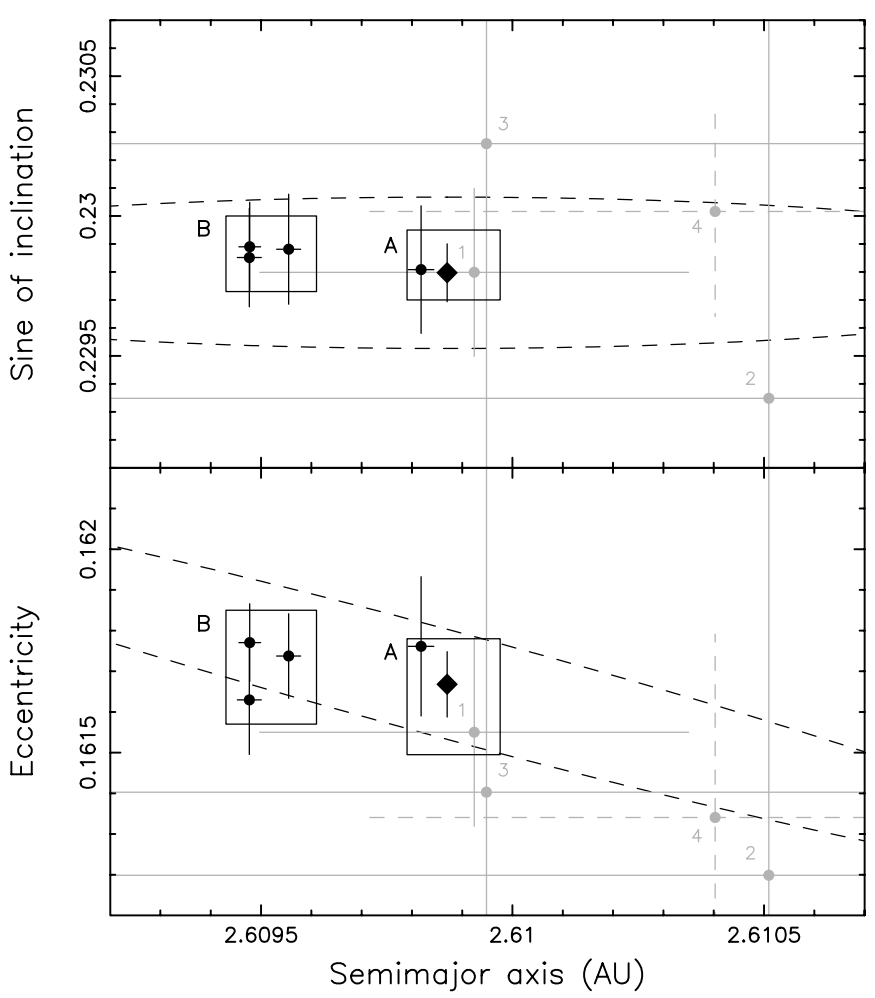

Figure 1. Synthetic proper elements of members in the Schulhof family: proper semimajor axis vs. proper sine of inclination (top) and proper semimajor axis vs. proper eccentricity (bottom). The error bars were obtained from integration of 20 clones starting in the uncertainty ellipsoid in the osculating orbital elements of the epoch. Black symbols and error bars show data for the multi-opposition asteroids, while gray symbols and error bars are for the single-opposition asteroids: 2007 EV68 (label 1), 2008 RA126 (label 2), and 2008 GW33 (label 3). We also show the solution for the nearby, but likely background asteroid 2008 EK72 (label 4 and dashed lines). The diamond shows the position of (2384) Schulhof. The boxes A and B indicate objects in the tight vicinity of (2384) Schulhof (box A) and (81337) 2000 GP36 (box B). The dashed ellipses indicate the theoretical borderline of the family if the fragments were ejected with an isotropic velocity field from (2384) Schulhof with a maximum speed of $5 \mathrm{~m} \mathrm{~s}^{-1}$, and at true anomaly $150^{\circ}$ and an argument of pericenter $210^{\circ}$ (see Zappalà et al. 1990).

(2384) Schulhof, where $d_{\text {prop }}$ is the standard three-dimensional distance in the space of proper orbital elements (e.g., Zappalà et al. 1990). This is less than the estimated escape velocity from this asteroid. Interestingly, the three multi-opposition asteroids in the cluster near (81337) 2000 GP36 enclosed in box B in Figure 1, i.e., (81337) 2000 GP36, 2001 UR193, and 2003 FK6, have approximately the same proper eccentricity and inclination as (2384) Schulhof, but their proper semimajor axes are $\sim 4 \times 10^{-4}$ AU smaller than that of (2384) Schulhof. The nearest multi-opposition object to (2384) Schulhof is 2009 EL11 from its own sub-cluster (box A in Figure 1).

The proper elements of single-opposition objects in the Schulhof family, namely, 2007 EV68, 2008 RA126, and 2008 GW33, are less accurate (gray symbols in Figure 1). While the nominal orbits have $d_{\text {prop }} \sim 10$ to $\sim 18 \mathrm{~m} \mathrm{~s}^{-1}$ from (2384) Schulhof, the uncertainty intervals comfortably overlap with the zone of the family. Moreover, the analysis of their past orbital evolution shows a good convergence to the Schulhof orbit. For that reason we believe that they are real family members.

\subsection{Numerical Backtracking of Orbits}

We now provide details on our aforementioned procedure for reconstructing the initial configuration and determining the 
age of the Schulhof family. We take into account (1) various, statistically equal past orbital evolutions starting near the nominal solution (namely, within $90 \%$ confidence level surface in six-dimensional space of osculating orbital elements) and (2) various past orbital evolutions with different, presently unconstrained, strengths of the Yarkovsky effect (e.g., Bottke et al. 2002, 2006). The first multitude of orbits-called geometrical clones-is due to the current orbital uncertainty as it results from a limited number and accuracy of observations. The second multitude of orbits-called Yarkovsky clones-is due to the incompleteness of the dynamical model where the effects of thermal forces were not taken into account. We used a simple model where the complete formulation of the Yarkovsky effect is represented with a drag-like acceleration producing a secular change, $d a / d t$, of the orbital semimajor axis. For a given size and heliocentric distance, we estimated the maximum value of $[d a / d t]_{\mathrm{m}}$ for the diurnal variant of the Yarkovsky effect (at zero obliquity; e.g., Vokrouhlický 1998, 1999). For reference, we note that $[d a / d t]_{\mathrm{m}} \sim 1.4 \times 10^{-4} \mathrm{AU} \mathrm{Myr}^{-1}$ for a $2 \mathrm{~km}$ size asteroid with $a=2.6 \mathrm{AU}$. Different Yarkovsky clones were assigned to have uniformly distributed values of the semimajor axis drift in the interval $\left(-[d a / d t]_{\mathrm{m}},+[d a / d t]_{\mathrm{m}}\right)$. This is based on the assumption that the orientation of spin axes is random in space. Since the orbit of (2384) Schulhof is much better determined than that of any other asteroid in the family, and its size is quite larger such that the Yarkovsky effect is smaller, we typically took hundreds of clones for this body. In the case of smaller asteroids in the cluster, we typically had thousands of clones.

We used the numerical code known as SWIFT_MVS (e.g., Levison \& Duncan 1994), in which we implemented orbital perturbation due to the drag acceleration (representing the Yarkovsky effect). We conducted backward integration of all clones to $1.5 \mathrm{Myr}$ before the present. The time step of the propagation was 3 days. We output state vectors of all particles every $10 \mathrm{yr}$. Gravitational perturbations of all planets were included. Their initial orbits were taken from JPL DE405 ephemeris file at epoch MJD55400. ${ }^{6}$

The strategy to estimate the age of the family is as follows (see Nesvorný \& Vokrouhlický 2006 for more details). At each past instant, for which we recorded the state vectors of all asteroid clones, we performed a large number of random selections (trials) of one clone for each asteroid and evaluated a "target function"

$$
\delta V=n a \sqrt{(\sin i \delta \Omega)^{2}+0.5(e \delta \varpi)^{2}} .
$$

Here $n a \approx 18.5 \mathrm{~km} \mathrm{~s}^{-1}$ is the orbital speed of asteroids in the cluster, and $\delta \Omega$ and $\delta \varpi$ are the dispersions of angles at some time in the past. If only two asteroids were considered, the dispersions of angles would just be their differences. When we considered clones of more than two objects, they were given by standard relations such as $(\delta \Omega)^{2}=\sum_{i j}\left(\delta \Omega_{i j}\right)^{2} / N$, with $\delta \Omega_{i j}$ denoting the differences of nodal longitudes between bodies $i$ and $j$, and $N$ being the number of such pairs. Note that for a collisionally born cluster of objects with an isotropic velocity field of dispersal velocity $V_{\text {disp }}$, the value of $\delta V$

\footnotetext{
6 The following trick was used to integrate backward with SWIFT_MVS using a positive time step: (1) the initial state vectors of both planets and asteroidal clones were modified according to the scheme

$\left(x, y, z ; v_{x}, v_{y}, v_{z}\right) \rightarrow\left(x, y,-z ;-v_{x},-v_{y}, v_{z}\right)$, and (2) the same

transformation was used at any time during the integration to obtain the past state vector and thus the correct Keplerian orbital elements.
}

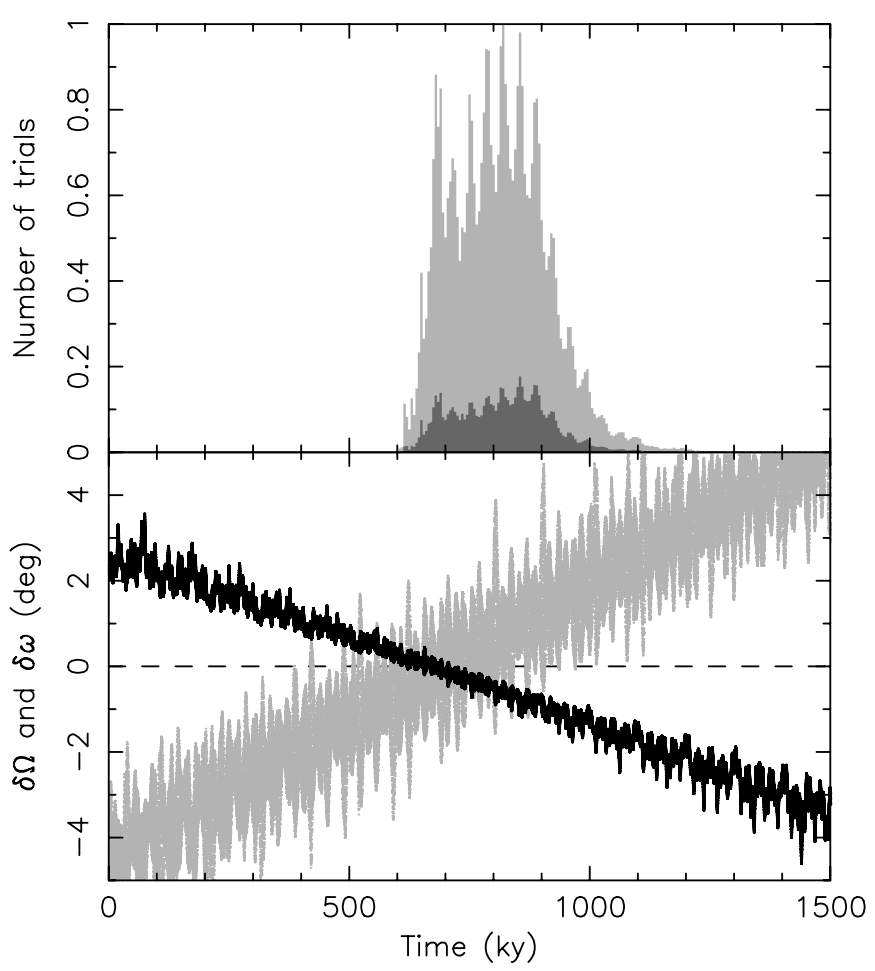

Figure 2. Convergence of secular angles of (81337) 2000 GP36 and (2384) Schulhof; the abscissa is the time in the past in kyr. Top: number of trials for which $\delta V<5 \mathrm{~m} \mathrm{~s}^{-1}$ (gray histogram) and $\delta V<2 \mathrm{~m} \mathrm{~s}^{-1}$ (black histogram) in Equation (1). Data were binned into $5 \mathrm{kyr}$ intervals and normalized to the maximum of the gray distribution. Bottom: example of a convergence at $\sim 685 \mathrm{kyr}$. The panel shows the difference $\delta \Omega$ in longitude of the node (black curve) and $\delta \omega$ of the argument of pericenter (gray curve) relative to Schulhof's orbit.

from Equation (1) is roughly $\sim \sqrt{2 / 3} V_{\text {disp }}$ (after averaging over the orbital phase). Since we expect $V_{\text {disp }}$ to be about the escape velocity from the parent body, the formation time of the family should be characterized with $\delta V \leqslant 5 \mathrm{~m} \mathrm{~s}^{-1}$. Note that the current $\delta V$ of orbits in the cluster around (81337) 2000 GP36 is $\sim 200 \mathrm{~m} \mathrm{~s}^{-1}$, roughly a factor of 40 larger. We thus used the backward-integrated orbits of clones for the Schulhof family members to seek time in the past-a "candidate age"-for which $\delta V \leqslant 5 \mathrm{~m} \mathrm{~s}^{-1}$. Because we have a multitude of statistically equivalent clones for each of the members, we can only characterize the age of the family with statistical means. More details of the family-reconstruction technique can be found in Nesvorný \& Vokrouhlický (2006) and Vokrouhlický \& Nesvorný (2008).

\subsubsection{Multi-opposition Asteroids in the 2000 GP36 Sub-cluster}

First, we consider multi-opposition objects in the vicinity of (81337) 2000 GP36 and investigate convergence of their orbits with (2384) Schulhof individually (the orbit of 2008 GW33 is too uncertain for our present analysis). We ran three simulations each of which (1) included clones of (2384) Schulhof and (2) separately included clones of (81337) 2000 GP36, 2001 UR193 and 2003 FK6. In particular, we took 30 geometric and 10 Yarkovsky clones of (2384) Schulhof, and 50 geometric and 60 Yarkovsky clones of each of the 2000 GP36 sub-cluster asteroids. We then considered a 750,000 trial selection of clones of the two asteroids in each run, which was close to all possible identifications $(300 \times 3000)$. Results are shown in Figures 2-4.

We can see that in all three cases the secular angles of (81337) 2000 GP36, 2001 UR193, and 2003 FK6 clones converge to 


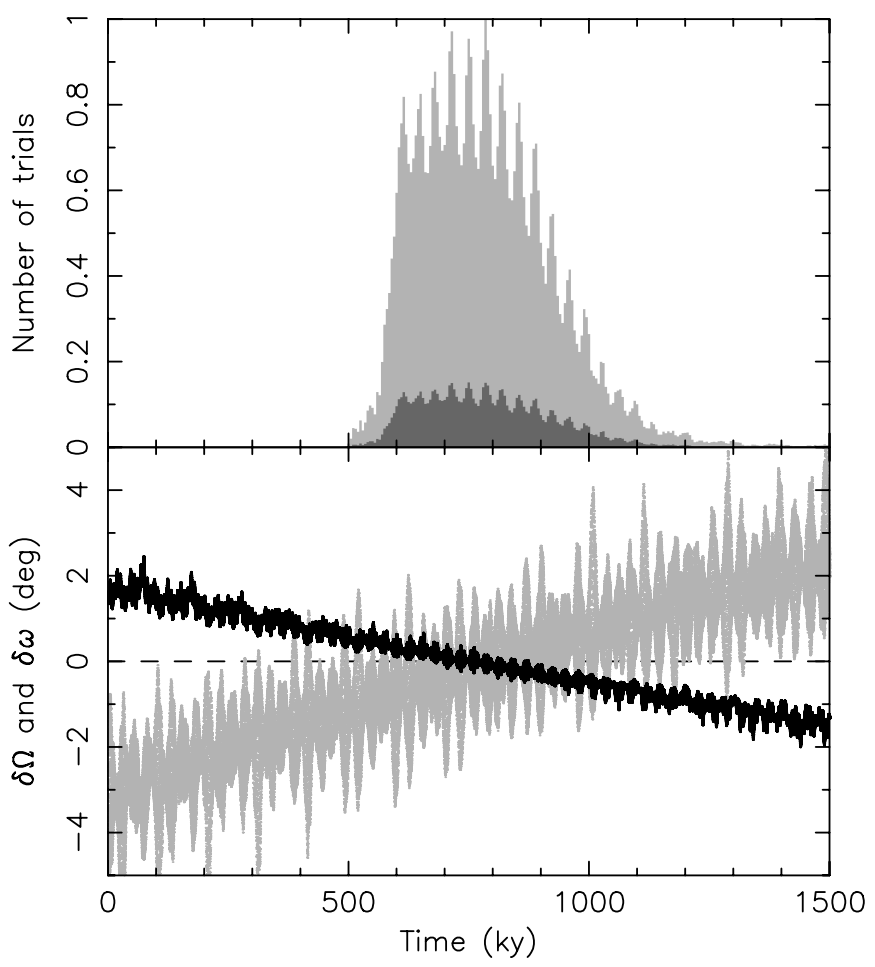

Figure 3. Convergence of secular angles of 2001 UR193 and (2384) Schulhof; the abscissa is the time in the past in kyr. Top: number of trials for which $\delta V<5 \mathrm{~m} \mathrm{~s}^{-1}$ (gray histogram) and $\delta V<2 \mathrm{~m} \mathrm{~s}^{-1}$ (black histogram) in Equation (1). Data were binned into $5 \mathrm{kyr}$ intervals and normalized to the maximum of the gray distribution. Bottom: example of a convergence at $\sim 741 \mathrm{kyr}$. The panel shows the difference $\delta \Omega$ in longitude of the node (black curve) and $\delta \omega$ of the argument of pericenter (gray curve) relative to Schulhof's orbit.

those of (2384) Schulhof some 600-800 kyr ago. The formal mean value and standard deviation of distributions for which $\delta V \leqslant 5 \mathrm{~m} \mathrm{~s}^{-1}$ in our simulations (shadow histograms in the upper panels of the figures) was (1) $810 \pm 100 \mathrm{kyr}$ for (81337) $2000 \mathrm{GP} 36$, (2) $780 \pm 140 \mathrm{kyr}$ for $2001 \mathrm{UR} 193$, and (3) $760 \pm 75 \mathrm{kyr}$ for 2003 FK6. At best, some $\sim 2 \%-4 \%$ of all trials satisfied $\delta V \leqslant 5 \mathrm{~m} \mathrm{~s}^{-1}$, showing that the convergence of the two orbits is rather robust. ${ }^{7}$ We find that the fundamental separation of the $2000 \mathrm{GP} 36$ sub-cluster from Schulhof's orbit is merely a difference in the proper semimajor axis. This difference is nearly fully responsible for the accumulated difference in secular angles today. For instance, considering the proper nodal frequency gradient $(\partial s / \partial a) \sim-25 \operatorname{arcsec} \mathrm{yr}^{-1} \mathrm{AU}^{-1}$ in the Schulhof family zone, we have $(\partial s / \partial a) \Delta a_{\mathrm{P}} T \sim+2.1$ for $\Delta a_{\mathrm{P}} \sim$ $-4 \times 10^{-4} \mathrm{AU}$ (Figure 1 ) and $T \simeq 750 \mathrm{kyr}$ (Figures $2-4$ ). ${ }^{8}$ Thus, the current $\simeq 2$ deg nodal difference is principally accounted for by proper $s$-frequency sensitivity to the semimajor axis difference. The same also applies to the pericenter longitude.

We also performed a secular-angle convergence simulation simultaneously taking into account the orbits of (2384) Schulhof

\footnotetext{
7 Note that the required threshold $\delta V \leqslant 5 \mathrm{~m} \mathrm{~s}^{-1}$ for the target function (1) is a rather severe constraint and it makes a number of clone identifications disqualified from good convergence. For instance a simple test run shows that geometric clones of (81337) 2000 GP36 acquire $\simeq \pm 0.3$ divergence of nodes some $800 \mathrm{kyr}$ ago. This is about five times more than $\simeq 0.05-0.1$ needed to satisfy the $\delta V \leqslant 5 \mathrm{~m} \mathrm{~s}^{-1}$ condition. Since even stronger divergence is observed for the longitude of pericenter, one cannot expect a larger success rate than $\sim 1 / 25 \simeq 4 \%$ in reconstruction of possible mutual configurations of asteroids (2384) Schulhof and (81337) 2000 GP36 in our simulation.

8 The Yarkovsky contribution to the nodal and pericenter drift of neighboring asteroids is not negligible but small. For instance, the node should have the maximum effect $\sim 0.5(\partial s / \partial a)(d a / d t) T^{2} \simeq 0$. 2 for $T=750 \mathrm{kyr}$.
}

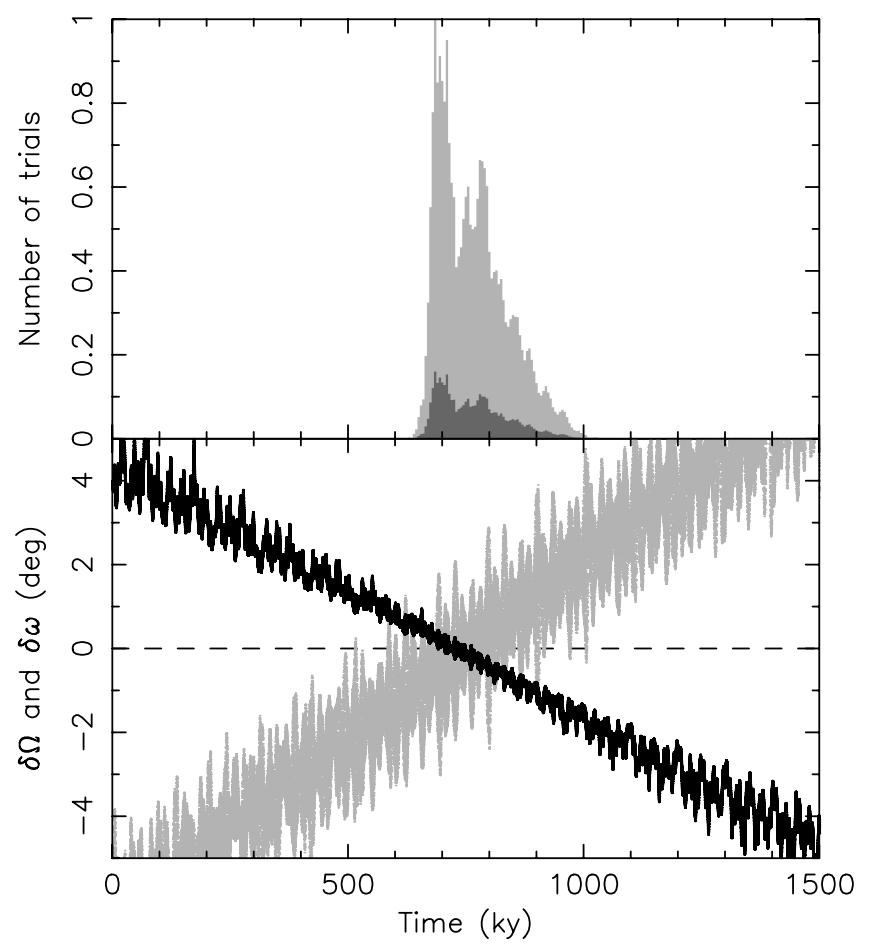

Figure 4. Convergence of secular angles of 2006 FK6 and (2384) Schulhof; the abscissa is the time in the past in kyr. Top: number of trials for which $\delta V<5 \mathrm{~m} \mathrm{~s}^{-1}$ (gray histogram) and $\delta V<2 \mathrm{~m} \mathrm{~s}^{-1}$ (black histogram) in Equation (1). Data were binned into $5 \mathrm{kyr}$ intervals and normalized to the maximum of the gray distribution. Bottom: example of a convergence at $\sim 712 \mathrm{kyr}$. The panel shows the difference $\delta \Omega$ in longitude of the node (black curve) and $\delta \omega$ of the argument of pericenter (gray curve) relative to Schulhof's orbit.

and all three multi-opposition bodies in the 2000 GP36 subcluster. In this case we had 20 geometric and 30 Yarkovsky clones for Schulhof, and 30 geometric and 40 Yarkovsky clones for the smaller asteroids. We tested 25 million trials. For every choice of trial identification we evaluated the target function (1). In this case, the pair-wise-constructed dispersion of the node and pericenter values had $N=6$ contributions. We obtained the age estimate of $780 \pm 100 \mathrm{kyr}$, which is similar to the age estimates obtained in the cases where objects were considered individually.

We also attempted to substantiate the previous analysis by monitoring the convergence of clones in the Cartesian space. This more ambitious goal has been successfully applied to asteroid pairs with ages less than $100 \mathrm{kyr}$ (e.g., Vokrouhlický \& Nesvorný 2008, 2009; Pravec et al. 2010). Figure 5 shows the result for (81337) 2000 GP36 and (2384) Schulhof (similar results were also obtained for the other two asteroids in the vicinity of 2000 GP36). We found that two clones can approach each other at the Hill radius distance of Schulhof $(\sim 3200 \mathrm{~km})$ and have very small relative velocity of approach $\left(\sim 9 \mathrm{~m} \mathrm{~s}^{-1}\right.$ or less) exactly in the interval of time between 700 and $900 \mathrm{kyr}$ when secular angles converge (Figure 2). However, the result is still not optimal: (1) the best solutions approach at a distance that is much larger than the physical size of objects and (2) only a very small fraction, $\sim 10^{-6}$, of trial identifications provides such solutions. There are several reasons why we cannot obtain better reconstruction of the separation history of 2000 GP36 sub-cluster objects from Schulhof. First, the age of the family is larger than in the asteroid pair case. Over such a long time span, the orbital divergence is relatively large and we would need to use orders of magnitude more clones and denser time 


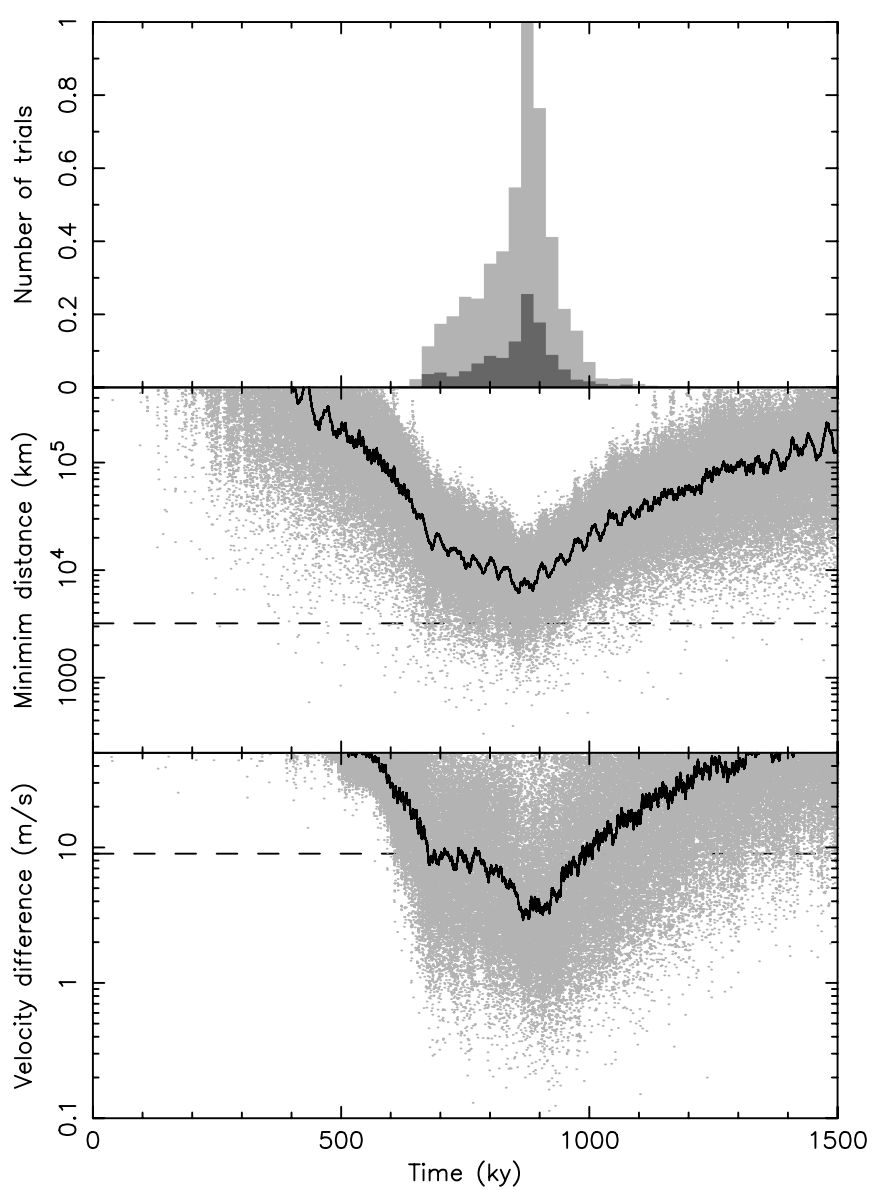

Figure 5. Convergence of the Cartesian space distance and relative velocity of clones for (81337) 2000 GP36 and (2384) Schulhof. Top: number of trials for which the clone distance was $<1.5$ times the Hill radius of Schulhof (gray histogram) and $<1$ Hill radius (black histogram) and the relative velocity of encounter was $<9 \mathrm{~m} \mathrm{~s}^{-1}$. Data were binned into $25 \mathrm{kyr}$ intervals and normalized to the maximum of the gray distribution. Middle: minimum distance between the trial clones at $10 \mathrm{yr}$ intervals (gray symbols). The black solid line is the mean value over a $5 \mathrm{kyr}$ running window and the dashed line is the estimated Hill radius for (2384) Schulhof ( $3200 \mathrm{~km})$. Bottom: relative encounter velocity between the trial clones that have minimum separation (middle panel). The dashed line is the maximum estimated escape speed from (2384) Schulhof $\left(\sim 9 \mathrm{~m} \mathrm{~s}^{-1}\right)$.

sampling to obtain a better solution. This surpasses the CPU and disk-quota limitations available to us. Second, such a fineresolution work will need a better dynamical model, in particular a more complete representation of the thermal forces.

\subsubsection{Asteroids in the Vicinity of Schulhof}

We now repeat the analysis from Section 2.3.1 for two asteroids in the immediate vicinity of (2384) Schulhof (bodies in box A in Figure 1): 2009 EL11 and 2007 EV68. While the first one resides on a multi-opposition orbit, the second one is on a single-opposition orbit. Both orbits are rather uncertain (Table 1). Still we try to determine the time when these objects separated from (2384) Schulhof using secular angles and a $\delta V<5 \mathrm{~m} \mathrm{~s}^{-1}$ condition. We used 30 geometric and 10 Yarkovsky clones for (2384) Schulhof, and 50 geometric and 60 Yarkovsky clones for the smaller members. Results are shown in Figures 6 and 7.

It can be noted that the condition $\delta V<5 \mathrm{~m} \mathrm{~s}^{-1}$ is satisfied for many clones already at the present epoch. This is because $\delta V<5 \mathrm{~m} \mathrm{~s}^{-1}$ roughly corresponds to nodal longitude difference $\delta \Omega \sim 0.05$ (and similarly for the pericenter longitude), which is larger than the $\sim 0.012$ difference between 2009 EL11 and

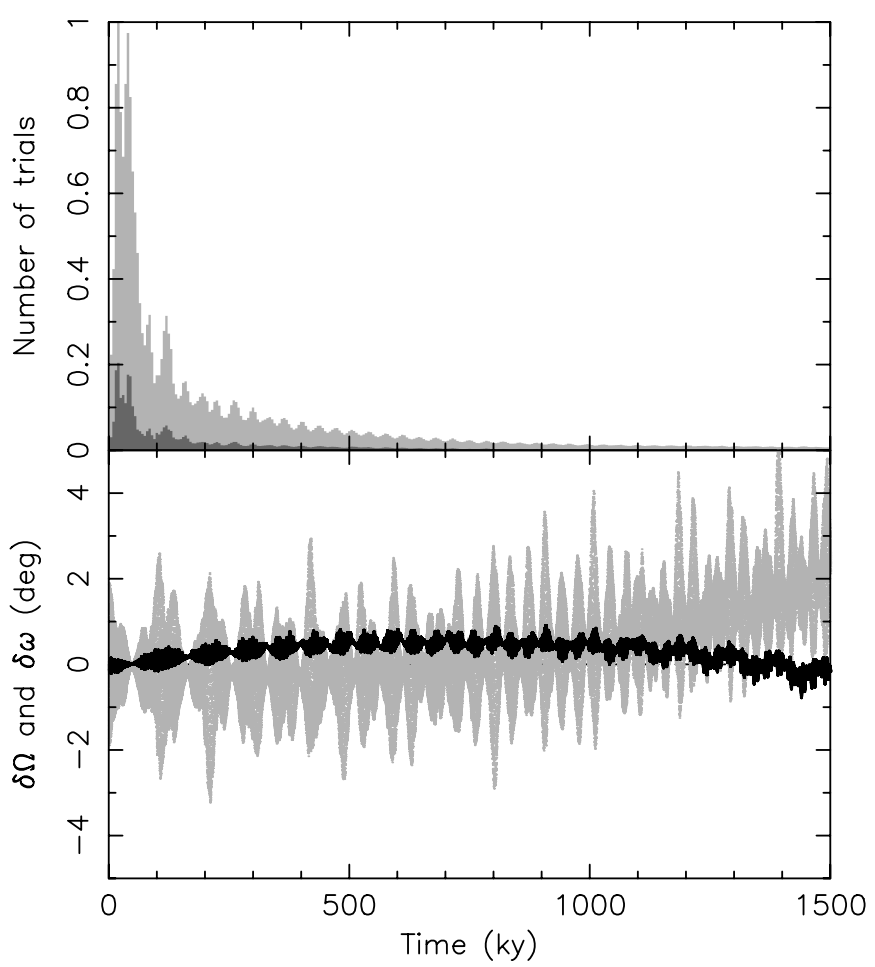

Figure 6. Convergence of secular angles of 2009 EL11 and (2384) Schulhof; the abscissa is the time in the past in kyr. Top: number of trials for which $\delta V<5 \mathrm{~m} \mathrm{~s}^{-1}$ (gray histogram) and $\delta V<2 \mathrm{~ms}^{-1}$ (black histogram) in Equation (1). Data were binned into $5 \mathrm{kyr}$ intervals and normalized to the maximum of the gray distribution. Bottom: example of a convergence at $\sim 42 \mathrm{kyr}$. The panel shows the difference $\delta \Omega$ in longitude of the node (black curve) and $\delta \omega$ of the argument of pericenter (gray curve) relative to Schulhof's orbit.

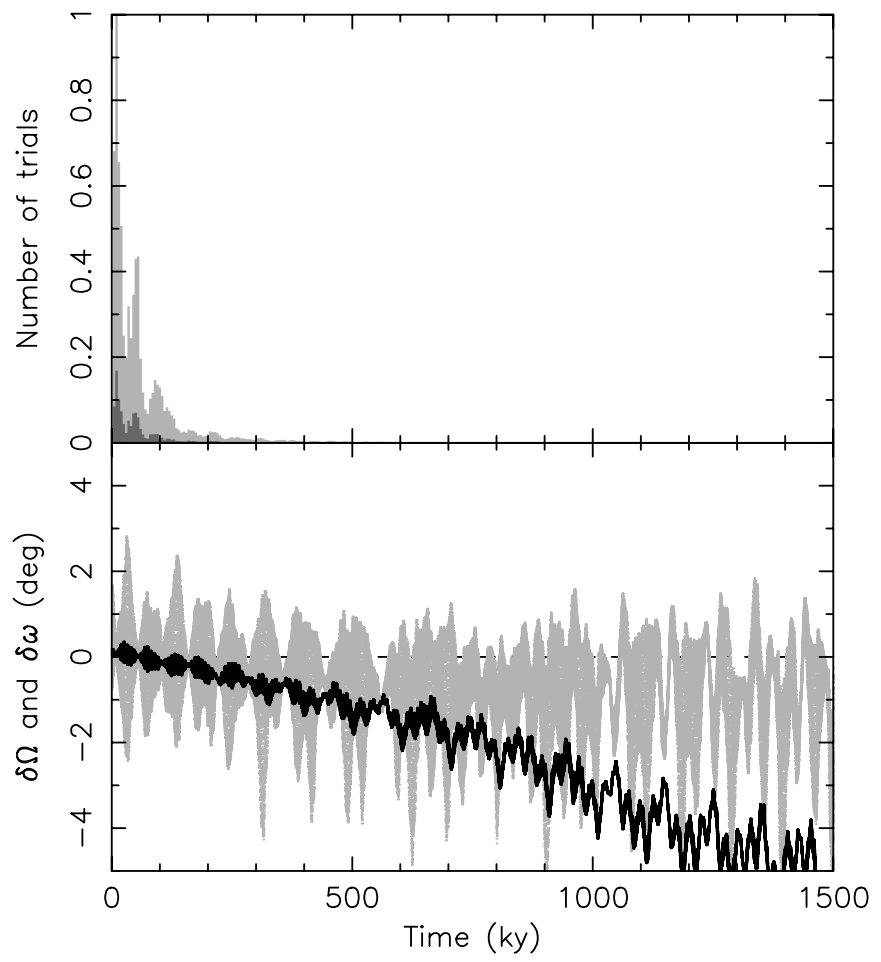

Figure 7. Convergence of secular angles of 2009 EL11 and (2384) Schulhof; the abscissa is the time in the past in kyr. Top: number of trials for which $\delta V<5 \mathrm{~m} \mathrm{~s}^{-1}$ (gray histogram) and $\delta V<2 \mathrm{~ms}^{-1}$ (black histogram) in Equation (1). Data were binned into $5 \mathrm{kyr}$ intervals and normalized to the maximum of the gray distribution. Bottom: example of a convergence at $\sim 48 \mathrm{kyr}$. The panel shows the difference $\delta \Omega$ in longitude of the node (black curve) and $\delta \omega$ of the argument of pericenter (gray curve) relative to Schulhof's orbit. 
Schulhof. Obviously, this does not mean that the two asteroids separated from each other in very recent past because their position in orbit differs. Our simulations show that the earliest time that both asteroids had a similar longitude in orbit was some $20 \mathrm{kyr}$ ago. Indeed, the distribution of trials satisfying $\delta V<5 \mathrm{~m} \mathrm{~s}^{-1}$ has a maximum some $50 \mathrm{kyr}$ ago and a long tail (Figure 6). While there are some solutions at $\geqslant 700 \mathrm{kyr}$ ago, they represent less than $0.1 \%$ of all cases. The currently available data thus indicate a much larger statistical likelihood that 2009 EL11 separated from (2384) Schulhof within the last $100 \mathrm{kyr}$. Data in Figure 7 show that the same result holds for asteroid 2007 EV68.

We also performed a test simulation without the Yarkovsky clones, including only 300 geometric clones for (2384) Schulhof and 3000 geometric clones for 2009 EL11. The goal was to see if the Yarkovsky clones do not artificially decrease the statistical likelihood of solutions with ages larger than $500 \mathrm{kyr}$. The results, however, look very similar to those in Figure 6. We also performed an analysis of clone convergence in Cartesian space for 2009 EL11 and a Schulhof "pair of objects." Again, the results are similar to those obtained from the analysis of secular angles, namely, they show statistical preference for solutions with ages less than $\sim 100 \mathrm{kyr}$.

\section{DISCUSSION AND CONCLUSIONS}

The real population of Schulhof family members has certainly yet to be characterized. Objects such as 2003 FK6 have $~ 19$ mag at the best opposition geometries, and the currently available surveys are fairly incomplete for such faint objects residing on orbits similar to Schulhof's. We can expect that many more small objects in the family will be discovered in the near future when the PanSTARRS and LSST surveys enter their full operational phase. On the other hand, the second largest member known today, (81337) 2000 GP36 has $~ 17.2$ mag at the best opposition geometries. The past survey programs have been quite successful in finding objects of this brightness. Thus, we do not expect that brighter/larger objects in the Schulhof family than 2000 GP36 will be discovered in the future. Given the $\sim 3$ mag difference between the first two largest objects, it is likely that the Schulhof family was created by a cratering event on (2384) Schulhof.

Asteroids from the neighborhood of (81337) 2000 GP36 seem to firmly establish the age of the family to be $780 \pm 100 \mathrm{kyr}$. Their displacement in the proper value of the semimajor axis, as compared to (2384) Schulhof, suggests a highly asymmetric ejection velocity field for the large fragments. To generate this offset, the largest ejection speed component would need to be in a transverse direction in the motion of the parent body. For a cratering event this is actually not a surprise, yet it has not been seen with such clarity before. This is because in old families created by cratering events, such as Massalia or Vesta, longterm dynamical processes erased information about the initial configuration of the fragments. Young asteroid families, such as the Schulhof family may thus serve as a suitable test case for numerical simulations of impact cratering events on asteroids.

Complications, however, arise from the orbital analysis of Schulhof family members in the immediate neighborhood of (2384) Schulhof. These objects seem to preferably separate from Schulhof within the last $100 \mathrm{kyr}$ (Figures 6 and 7, and discussion in Section 2.3.2). This situation requires some explanation.

Standard hypothesis. The problem may stem from the large uncertainty of the Schulhof sub-cluster orbits. For instance, in the case of the 2009 EL11 and the Schulhof pair solutions with a convergence of $\sim 700 \mathrm{kyr}$ ago formally exist (though their collective statistical weight is very small). Future orbit improvements of 2009 EL11, 2007 EV68, and 2008 RA126 (recoveries in the last two cases) will be needed to test this possibility.

Alternate hypotheses. However, as shown above, 2009 EL11, 2007 EV68, and 2008 RA126 may have separated from Schulhof hundreds of thousands of years after the formation of the family. One possibility is a second cratering event on (2384) Schulhof. However, estimating that at least a $\sim 200 \mathrm{~m}$ impactor would have been needed to produce the second component in the Schulhof family, we do not find this event very likely. From Bottke et al. (2005), we estimate a characteristic timescale of 10-20 Myr for such an impact to occur. It is thus unlikely that a second impact happened $\sim 600 \mathrm{kyr}$ after the formation of the family. The fast rotation rate of (2384) Schulhof may hint at another possibility. Specifically, the family-forming event $\sim 700 \mathrm{kyr}$ ago probably left this asteroid in a state of very fast rotation, possibly close to the rotational fission limit, and/or might have produced one or more satellites around Schulhof (e.g., Durda et al. 2004). Later, fission instability of Schulhof, due to acceleration of its rotation by thermal torques, might have occurred and at the same time destabilized the satellite system. Such an event would have thus created additional members of the family on heliocentric orbits very close to that of (2384) Schulhof. While interesting, this scenario also suffers a probability caveat. Having currently three small members in the Schulhof sub-clump implies that, once corrected for incompleteness of the observations, there are many more yet to be discovered. The total number of asteroids in the immediate vicinity of Schulhof would thus largely exceed possible objects from satellite system dispersion.

Further considerations. Interestingly, we noticed that the ADR4 catalog of SDSS contains two observations of (81337) 2000 GP36. Taking a mean value from these observations we get $\mathrm{PC}_{1} \simeq 0.01 \pm 0.04$ for this object. This is an indication of a significantly bluer spectrum than that of (2384) Schulhof (Section 2): (81337) 2000 GP36 resides at the outskirts of the $\mathrm{S}$ complex in the $\mathrm{PC}_{1}$ versus $\mathrm{PC}_{2}$ plane of color indexes, which could indicate $\mathrm{Sq}$ or even $\mathrm{Q}$ classification (e.g., Parker et al. 2008). The fact that the central (largest) asteroid in the family is $\mathrm{S}$ type, while some smaller members may be Sq or Q types, recalls the case of other young asteroid families such as the Datura family (e.g., Nesvorný et al. 2006a; Vokrouhlický et al. 2009). In that case, Takato (2008), Mothé-Diniz \& Nesvorný (2008), and Willman et al. (2010) found that (1270) Datura has S type classification, but at least two smaller members-2003 CL5 and 2001 WY35-have Sq and Q type classifications. If these results are confirmed, they may bear interesting hints about asteroid surfaces. For example, these trends can be explained if largest bodies in families retain significant debris from the initial fragmentation event.

Important constraints on the Schulhof family origin will be provided by the astrometric observation of 2000 EL11 during its next opposition in 2011 October. Having 21 mag in visual band, this observation should be easily done. The present right ascension uncertainty of the asteroid position is $\sim 2 \operatorname{arcmin}$. Thus if routine astrometric data with an uncertainty of an arcsecond or so will be obtained, the uncertainty in the semimajor axis solution may improve by more than two orders of magnitude (note that the orbital arc bracketed with the first and the last observation will nearly double). With this improvement of the 2009 EL11's orbit it might be possible to resolve the problem about the time of its separation from (2384) Schulhof. We thus encourage observers to carry out these observations. 
This research was supported by NASA's Planetary Geology and Geophysics Program, Czech Grant Agency (grant 205/08/0064), and the Research Program MSM0021620860 of the Czech Ministry of Education. We thank F. Bernardi for providing orbital details for the single-opposition asteroid 2008 RA126.

\section{REFERENCES}

Bottke, W. F., Durda, D. D., Nesvorný, D., Jedicke, R., Morbidelli, A., Vokrouhlický, D., \& Levison, H. F. 2005, Icarus, 179, 63

Bottke, W. F., Vokrouhlický, D., Rubincam, D. P., \& Brož, M. 2002, in Asteroids III, ed. W. F. Bottke et al. (Tucson, AZ: Univ. Arizona Press), 395

Bottke, W. F., Vokrouhlický, D., Rubincam, D. P., \& Nesvorný, D. 2006, Ann. Rev. Earth Planet. Sci., 34, 157

Chapman, C. R. 2004, Ann. Rev. Earth Planet. Sci., 32, 539

Ditteon, R., Bixby, A. R., Sarros, A. M., \& Waters, C. T. 2002, Minor Planet Bull., 29, 69

Durda, D. D., Bottke, W. F., Enke, B. L., Merline, W. J., Asphaug, E., Richardson, D. C., \& Leinhardt, Z. M. 2004, Icarus, 170, 243

Espy, A. J., Dermott, S. F., Kehoe, T. J. J., \& Jayaraman, S. 2009, Planet. Space Sci., 57, 235

Farley, K. A., Vokrouhlický, D., Bottke, W. F., \& Nesvorný, D. 2006, Nature, 439, 295

Gaffey, M. J. 2010, Icarus, 209, 564

Ivezić, Z., et al. 2001, AJ, 122, 2749

Jedicke, R., Nesvorný, D., Whiteley, R., Ivezić, Ž., \& Jurić, M. 2004, Nature, 429, 275

Knežević, Z., Lemaitre, A., \& Milani, A. 2002, in Asteroids III, ed. W. F. Bottke et al. (Tucson, AZ: Univ. Arizona Press), 603

Lazzaro, D., et al. 1999, Icarus, 142, 445
Levison, H. F., \& Duncan, M. J. 1994, Icarus, 108, 18

Mothé-Diniz, T., \& Nesvorný, D. 2008, A\&A, 486, L9

Mothé-Diniz, T., Roig, F., \& Carvano, J. M. 2005, EAR-A-VARGBDET-5MOTHEFAM-V1.0, NASA Planetary Data System

Nathues, A. 2010, Icarus, 208, 252

Nesvorný, D., Bottke, W. F., Dones, L., \& Levison, H. F. 2002, Nature, 417, 720

Nesvorný, D., Bottke, W. F., Levison, H. F., \& Dones, L. 2003, ApJ, 591, 486

Nesvorný, D., Bottke, W. F., Vokrouhlický, D., Sykes, M. V., Lien, D. J., \& Stansberry, J. 2008, ApJ, 679, L143

Nesvorný, D., \& Vokrouhlický, D. 2006, AJ, 132, 1950

Nesvorný, D., Vokrouhlický, D., \& Bottke, W. F. 2006a, Science, 312, 1490

Nesvorný, D., Vokrouhlický, D., Bottke, W. F., \& Sykes, M. V. 2006b, Icarus, 181,107

Nesvorný, D., et al. 2006c, AJ, 132, 582

Novaković, B. 2010, MNRAS, 407, 1477

Parker, A., Ivezić, Z., Jurić, M., Lupton, R., Sekora, M. D., \& Kowalski, A. 2008, Icarus, 198, 138

Pravec, P., \& Vokrouhlický, D. 2009, Icarus, 204, 580

Pravec, P., et al. 2010, Nature, 466, 1085

Rożek, A., Breiter, S., \& Jopek, T. J. 2011, MNRAS, 412, 987

Takato, N. 2008, ApJ, 685, L161

Vernazza, P., Binzel, R., Birlan, M., Fulchignoni, M., \& Rossi, A. 2009, Nature, 458, 993

Vokrouhlický, D. 1998, A\&A, 335, 1093

Vokrouhlický, D. 1999, A\&A, 344, 362

Vokrouhlický, D., \& Nesvorný, D. 2008, AJ, 136, 280

Vokrouhlický, D., \& Nesvorný, D. 2009, AJ, 137, 111

Vokrouhlický, D., Nesvorný, D., \& Bottke, W. F. 2008, ApJ, 672, 696

Vokrouhlický, D., et al. 2009, A\&A, 507, 495

Willman, M., Jedicke, R., Moskovitz, N., Nesvorný, D., Vokrouhlický, D., \& Mothé-Diniz, T. 2010, Icarus, 208, 758

Zappalà, V., Cellino, A., Farinella, P., \& Knežević, Z. 1990, AJ, 100, 2030 